\title{
Definitions/Epidemiology/Risk Factors for Sexual Dysfunction
}

\author{
Ronald W. Lewis, MD, ${ }^{*}$ Kerstin S. Fugl-Meyer, PhD, ${ }^{\dagger}$ Giovanni Corona, MD, ${ }^{\ddagger}$ Richard D. Hayes, PhD, ${ }^{\S}$ \\ Edward O. Laumann, PhD, ${ }^{\uparrow}$ Edson D. Moreira Jr., MD, PhD, ${ }^{* \star}$ Alessandra H. Rellini, PhD, ${ }^{+\dagger}$ and \\ Taylor Segraves, MD, $\mathrm{PhD}^{\ddagger \ddagger}$ \\ *Surgery/Urology, Medical College of Georgia, Augusta, GA, USA; ${ }^{\dagger}$ Andrology and Sexual Medicine, Karolinska \\ University Hospital Huddinge, and Medicine, Karolinska Institutet, Stockholm, Sweden; ₹Sexual Medicine and Andrology, \\ Physiopathology, University of Florence, Florence, Italy and Endocrinology, Maggiore-Bellaria Hospital, Bologna, Italy; \\ \$Health Services and Population Research, King's College London, London, UK; "Sociology, University of Chicago, \\ Chicago, IL, USA; **Oswaldo Cruz Foundation, Gonçalo Moniz Research Center, Salvador, Bahia, Brazil; ${ }^{\dagger}$ Psychology, \\ University of Vermont, Burlington, VT, USA; 抑Psychiatry, Case Western Reserve School of Medicine, Cleveland, Ohio, \\ USA
}

DOI: $10.1111 / \mathrm{j} .1743-6109.2010 .01778 . x$

\section{A B S T R A C T}

Introduction. Accurate estimates of prevalence/incidence are important in understanding the true burden of male and female sexual dysfunction and in identifying risk factors for prevention efforts. This is the summary of the report by the International Consultation Committee for Sexual Medicine on Definitions/Epidemiology/Risk Factors for Sexual Dysfunction.

Aim. The main aim of this article is to provide a general overview of the definitions of sexual dysfunction for men and women, the incidence and prevalence rates, and a description of the risk factors identified in large populationbased studies.

Methods. Literature regarding definitions, descriptive and analytical epidemiology of sexual dysfunction in men and women were selected using evidence-based criteria. For descriptive epidemiological studies, a Prins score of 10 or higher was utilized to identify population-based studies with adequately stringent criteria. This report represents the opinions of eight experts from five countries developed in a consensus process and encompassing a detailed literature review over a 2-year period.

Main Outcome Measures. The study aims to provide state-of-the-art prevalence and incidence rates reported for each dysfunction and stratified by age and gender. Expert opinion was based on the grading of evidence-based medical literature, widespread internal committee discussion, public presentation, and debate.

Results. A wealth of information is presented on erectile dysfunction, its development through time, and its correlates. The field is still in need of more epidemiological studies on the other men's sexual dysfunction and on all women's sexual dysfunctions.

Conclusions. A review of the currently available evidence from epidemiological studies is provided. Lewis RW, Fugl-Meyer KS, Corona G, Hayes RD, Laumann EO, Moreira ED Jr., Rellini AH, and Segraves T. Definitions/Epidemiology/Risk Factors for Sexual Dysfunction. J Sex Med 2010;7:1598-1607.

Key Words. Sexual Dysfunction Definition for Men and Women; Descriptive and Analytical Sexual Dysfunction; Epidemiology; Prevalence of Sexual Dysfunctions; Incidence of Sexual Dysfunction; Risk Factors for Sexual Dysfunction; Erectile Dysfunction; Premature Ejaculation 


\section{Introduction}

$\mathrm{T}$ here is a need to recognize that the definitions for sexual dysfunctions may be different for epidemiological studies vs. those needed for clinical applications. Epidemiology attempts to describe the incidence and prevalence of a condition in a community or population. For descriptive epidemiology studies in sexual medicine, incidence and prevalence for symptoms in a given population are explored. For clinical definitions, further expansions such as duration, severity, and bother may need to be part of the defined dysfunction evaluated and treated. For the most part, this committee presents per se definitions. Descriptive and analytical epidemiological studies of various sexual dysfunctions in men and women backed by evidence-based medicine were examined. The work of these authors on the committee for definitions, classifications, epidemiology of sexual dysfunction is summarized.

\section{Methods}

The eight committee members from five countries collected pertinent peer-reviewed publications and evidence-based book chapters prior to the International Consultation in Paris. These publications were graded at the $\mathrm{I}_{\mathrm{b}}$ hierarchical level using evidence-based criteria [1]. The literature was located through data bases, such as Medline, Scopus, PUB MED, and Psych Info from 2002 to 2009 using key word including sexual dysfunction in general, each of the specific dysfunctions defined in the chapter, women/female, men/male, epidemiology, sexual interest, sexual desire, sexual arousal, erection, penile erection, sexual satisfaction, vaginal lubrication, diagnostic criteria, psychological \& psychiatric factors, psychiatric drug effect, psychosocial comorbidity, sexual disorders, and sexual dysfunction. The previous chapter [2] also served as source of publications but some of these references were deleted because on re-examination, they did not reach high evidencebased standards. Selected articles and book chapter reference lists were also searched for further pertinent and evidence-based data. The table of contents of several journals were also searched for pertinent articles including but not limited to Fournal of Sexual Medicine, Fournal of Urology, Urology, British fournal of Urology International, International Fournal of Impotence Research, Archives of Sexual Behavior, Fournal of Andrology, and International Fournal of Andrology. All descriptive epide-
Table 1 Criteria for the methodical quality assessment of prevalence studies-one point for yes to lower case

External validity

Source population

(a) Does the method to select and invite participants result in a study population that covers the complete population or a random sample?

Description of the eligibility criteria

(b) Is the age range specified?

(c) Are inclusion and exclusion criteria specified?

Participants and nonresponders

(d) Is the response rate $>70 \%$, or is the information on nonresponders sufficient to make inference on the representativeness of the study population?

Description of the study period

(e) Is the study period specified?

Description of the study population

(f) Are important population characteristics* ${ }^{*}$ specified?

Internal validity

Data collection

(g) Are the data prospectively collected?

Measurement instrument (questionnaire, interview, additional)

(h) Is the measurement instrument validated?

(i) Is the period covered by the measurement instrument specified?

Definition of diseases ${ }^{\dagger}$

(j) Is a definition of the disease stated?

Reported prevalences

(k) Are age-specific and gender-specific prevalences reported?

(l) Are possible correlates of disease ${ }^{\dagger}$ reported?

Informatively

(m) Is the method of data collection properly described (interview, questionnaire, additional measurement)?

(n) Are the questions and answer possibilities stated?

(o) Are the reported prevalence rates reproducible?

${ }^{*}$ Two or more of: (i) age distribution; (ii) relevant comorbidity; (iii) lifestyle factors (e.g. smoking and alcohol consumption); and (iv) socio-economic data (e.g., income, education level, marital status).

${ }^{\dagger}$ Disease equals erectile dysfunction in this interview.

miological articles were graded using the 15-point scaled Prins et al. (see Table 1), and were included only if reaching a score of 10 points [3]. A consensus of the committee was reached in Paris during the consultation to determine what data would be included in the final chapter. Definitions were updated from the previous chapter by committee consensus. Generally, the references on descriptive epidemiology were read by two or more of the committee members in order to be reasonably sure of their epidemiological validity.

\section{Definitions}

Based upon the committee's deliberation, the following definitions are given for sexual disorders in men and women. These do not generally separate organic- from psychogenic-caused dysfunctions. For clinical reference, etiology causing the disorders may be clarified by further diagnostic studies but the two categories of organic and psychological are not mutually exclusive. 
Sexual interest/desire dysfunctions are diminished or absent feeling of sexual interest or desire, absent sexual thoughts or fantasies, and a lack of responsive desire; motivations (here defined as reason-incentives) for attempting to become sexually aroused are scarce or absent. This definition is applicable to this sexual disorder in men and women.

Sexual arousal disorders in women are divided into three subtypes. Genital sexual arousal dysfunctions are absent or impaired genital sexual arousal; self-report may include minimal vulval swelling or vaginal lubrication from any type of sexual stimulation and reduced sexual sensation from direct stimulation of genitalia. Subjective excitement still occurs from nongenital sexual stimuli. Subjective sexual arousal dysfunction is the absence of or markedly diminished feelings of sexual arousal (sexual excitement and sexual pleasure), from any type of sexual stimulation. Vaginal lubrication or other signs of physical response still occur. Combined genital and subjective arousal dysfunction is the absence of markedly diminished feelings of sexual arousal (sexual excitement and sexual pleasure) from any type of sexual stimulation as well as complaints of absent or impaired genital sexual arousal (vulval swelling, lubrication). Erectile dysfunction (ED) is the major sexual arousal disorder in men. It is defined as a consistent or recurrent inability of a man to obtain and/or maintain penile erection sufficient for sexual activity. A 3-month duration of these symptoms is accepted for establishing the diagnosis except for some instances of trauma or surgically induced ED.

Persistent genital arousal dysfunction is spontaneous, intrusive, and unwanted genital arousal (i.e., tingling, throbbing, pulsating) in the absence of sexual interest and desire. Any awareness of subjective sexual arousal is typically but invariably unpleasant. The arousal is unrelieved by one or more orgasms and the feeling or arousal persists for hours or days.

A new definition for premature ejaculation that was developed by the 2007 International Society for Sexual Medicine expert panel is the following: Life-long premature ejaculation is ejaculation which always or nearly always occurs prior to or within about 1 minute of vaginal penetration, and the inability to delay ejaculation on all or nearly all vaginal penetrations, and negative personal consequences such as distress, bother, frustration, and/or the avoidance of sexual intimacy [4]. The expert panel concluded that there was insufficient evidence to propose definitions for acquired premature ejaculation. Anejaculation is the absence of ejaculation at the time of orgasm.

Orgasmic dysfunction in men or women is the inability to achieve an orgasm, markedly diminished intensity of orgasmic sensations, or marked delay of orgasm during any kind of sexual stimulation. Individuals with this disorder report (high) sexual arousal/excitement. In men, orgasm dysfunction can occur together with ejaculatory dysfunction (for example, delayed orgasm and delayed ejaculation). Delayed ejaculation, which must be distinguished from retrograde ejaculation, is the unwanted delay of ejaculation during sexual activities.

Dyspareunia is persistent or recurrent pain during sexual activity. In women, this persistent or recurrent pain occurs with attempted or complete vaginal entry and/or penile vaginal intercourse.

Vaginismus is a persistent or recurrent difficulty of the women to allow vaginal entry of a penis, a finger, and/or any object, despite the women's expressed wish to do so. There is often (phobic) avoidance in anticipation/fear of pain.

For clinical studies and relevance to clinical situations, various descriptors are recommended for the various sexual dysfunction in women and men. They include degree of distress scales, lifelong or acquired status, situational or generalized occurrence, and other relevant conditional states.

Definitions gain applicability for comparative studies by including the degree of dysfunction. The degree of severity of sexual problems can be achieved through the administration of validated indices, such as the International Index of Erectile Function (IIEF) [5] for men and the Female Sexual Function Index [6] for women. There is now good evidence that a single item question can produce very different prevalence estimates than a validated multi-item scale when measuring sexual dysfunction in men and women [7]. Useful trichotomized scales including no dysfunction (never), mild dysfunction (hardly ever, rather rarely), and manifest dysfunction (rather often, often, nearly always, and always) have been suggested as a useful scale to classify severity [8].

\section{Incidence}

There is still clearly a need for more longitudinal studies for all of the sexual dysfunction in men and women in order to obtain more accurate incidence data. The sexual disorder that has the most 
evidence-based studies for incidence is ED. Five population-based studies produce incidence data on ED: two from the United States $[9,10]$, one from Brazil [11], one from the Netherlands [12], and one from Finland [13,14]. Details of these studies are presented in tabular form in the main book chapter published by this committee [15]. The crude incidence for ED is very similar in the American studies: 26 [9] and 28 [10] cases/1,000 man-years. A similar crude incidence of 28 cases/ 1,000 man-years was also presented in the study from the Netherlands [12], for clinically relevant ED at a mean follow-up of 2.1 years. The time of follow-up for the American studies were 8.8 years [9] and 2 years [10], respectively. However, in the study from the Netherlands [12], in the same group studied at a later period of time (almost doubling the follow-up time at 4.2 years), the crude incidence fell to 14 cases/1,000 man-years for clinically relevant ED. Age-specific incidence rates increased with aging for all of the studies. The more recent American study [10] also described crude incidence rate for low libido, ejaculatory dysfunction, perceived sexual problems and low sexual satisfaction, with the following case incidence/1,000 man-years of 35, 24, 16, and 37, respectively. The crude incidence of ED from Finland [13,14] and from Brazil [11] is higher, respectively, 39 and 66 cases/1,000 man-years. The incidence of sexual dysfunction in women is poorly established by evidence-based medicine reports leading the committee to recommend that there is a need for more incidence studies, from all regions of the world, for sexual dysfunction other than ED in men and all sexual dysfunctions in women. However, crude incidences for manifest low desire in women across wide age ranges of 18-74 years varied greatly from $10 \%$ in three studies to $19 \%$ in a Danish study to $35-41 \%$ in three other studies [15]. Greater desire levels were found in younger women, physically active, unmarried, with no children under 15 years, in good physical and mental health, and without medication.

\section{Prevalence}

Prevalence rates for sexual dysfunctions are strongly supported by evidence-based literature. Reasonably valid studies on prevalence of women's sexual dysfunctions were obtained from 18 descriptive epidemiological studies including seven from Europe, four from the United States, three from Australia, and one each from Canada,
Iran, Morocco, and Puerto Rico. For male sexual dysfunctions other than ED, there were 31 papers that were deemed meeting evidence-based literature standards to describe the incidence of these disorders. For ED, there were 59 studies from around the world. These are all tabulated in the main book chapter [15]. There is a variation in the prevalence rate reported because of different age groups reported, differences in definitions used to describe the dysfunctions, data selection, data collection, length of time the dysfunction was present, the degree of severity of the dysfunction, and possible socio-cultural differences. The prevalence of all sexual dysfunction for the most part increases as men and women become older. There is, by and large, reasonable valid descriptive epidemiological data indicating that about $40-45 \%$ of adult women and $20-30 \%$ of adult men have at least one manifest sexual dysfunction (rather often, often, nearly always, and always).

For women, the prevalence of manifest low levels of sexual interest varies between $17 \%$ and $55 \%$. Manifest low levels of sexual desire increase with age, with approximately $10 \%$ women up to the age of 49 years having a low level of desire, the prevalence then doubling to $22 \%$ in those aged $50-65$ years and again doubling to $47 \%$ in the 66-74-year-olds. Generally, arousal and lubrication problems are prevalent in $8-15 \%$, although three studies have reported this at a higher level of $21-28 \%$ in sexually active women. Some studies have evidence that with increasing age, in particular age greater than 50 years, lubrication insufficiency becomes more prevalent. For manifest orgasmic dysfunction, there is a great variability in reported prevalence rates. In the United States, Australia, Canada and Sweden, the prevalence of manifest orgasmic dysfunction is about $16-25 \%$ in $18-74-$ year-old women. Most of the studies have not reported an age dependency except in three, one study from Australia, one from Iran, and one from the United States [15]. Other studies from five areas report low rates (in the teens) for manifest orgasm dysfunction. In two reports from Nordic countries, where identical methodology was used, more than $80 \%$ of all sexually active women age $18-74$, independent of age, report some degree of orgasmic dysfunction [15]. The prevalence of manifest dyspareunia has been reported as low as $1 \%$ in Australian women yet $14-27 \%$ in seven other reports from around the world [15].

For men, dysfunction of sexual desire is generally much less prevalent than dysfunction of interest. The population level of sexual interest appears 
quite stable for the late teens up to about 60 years of age, after which it decreases markedly. Twentyfour epidemiologically valid reports that included men's interest and/or desire are included in tabular form in the book chapter [15]. In five different reports from around the world (Australia, Argentina, Southeast Asia, Korea, and Italy), the prevalence of desire/interest dysfunction, without any pronounced aging effect, of about $25 \%$ was reported for 16-59- and 40-80-year-old men. In general, studies published since the last consultation (2004) for prevalence of desire/interest have been reported for 40-80-year-olds without age adjustment. These reports range from $8 \%$ to $18 \%$ around the world except for the five studies mentioned above.

The prevalence rate for ejaculatory disturbances fulfilling valid Prins criteria have been found in 22 investigations that are tabulated in the main book chapter [15]. The prevalence varies from $8 \%$ to $30 \%$ for all age groups covered by the tabulated data for early or premature ejaculations. Two exceptions to this were a prevalence of $55 \%$ in 50-59-year-old men in one U.S. study [16], and a very low prevalence in 18-77-year-olds surveyed in London with a rate of 4\% [17]. More recent surveys reported in table 5 in the full chapter [15] have shown higher prevalence rates for early or premature ejaculation from around the world, particularly from Asia and Latin America. One exception to this, however, was a study from China in which the rate of persistent premature ejaculation was $8 \%$ [18] ( $\geq 2$ months) with a rate of $69 \%$ of any reoccurrence of this complaint within the previous year. A more recent report from Ahn et al. from Korea, found that in 1,570 men between 40 and 79 years of age, there was a prevalence rate of $25 \%$ for inability to control ejaculation time. Ejaculation time was further broken down as self-reported intervaginal ejaculation latency time of 2 minutes producing a prevalence rate of $11 \%$ and for 5 minutes, $33 \%$, both increasing significantly with aging [19]. Studies tabulated in the main book chapter show that the prevalence of delayed ejaculation is less studied with data from six reports in which a prevalence rate from $1 \%$ to $10 \%$ was estimated. One of the problems of surveys regarding early ejaculation is the inconsistency of how the condition is defined. This is discussed in the main book chapter [15]. In the book chapter from the previous edition, three studies were reported for an incidence rate for orgasm problems for 1-8\%. Since the last consultation, 13 of 23 published prevalence data for male dysfunctions other than ED have reported orgasm problems analyzed in their surveys. Most studies reported a prevalence rate in the range of $12-19 \%$ [15].

The prevalence of genital pain in men during sexual intercourse has only been fragmentarily studied. Rates from $3 \%$ to $6 \%$ have been reported in seven studies and prevalence rates of $1-2 \%$ in six studies. This is tabulated in the full book chapter [15]. Again, the study conducted in China reported a difference between occurring anytime within the previous year vs. a problem that persisted longer than 2 months with occurrence rates of $25 \%$ and $2 \%$, respectively [19].

ED is the sexual dysfunction for which we have the strongest evidence-based prevalence data that fulfill the stringent criteria outlined in Table 1. Fifty-nine studies from around the world regarding prevalence of ED from 1993 to 2009 are summarized in the full book chapter [15]. There are five studies from Asia, four from Australia, 30 from Europe, seven each from South/Central America and from North America, and six studies classified as Worldwide representing data collected from different regions of the world and published as a single article. It was difficult to compare these 59 studies. The definition used for ED shows a great variation. Methods of collecting the data varied from study to study. Time periods covered by the questions about $\mathrm{ED}$ varied from a few months to 1 year, and some of the studies did not specify a time period for ED. Of the 59 studies included in the full chapter tables [15], only eight utilized less than 500 participants. All of the studies that stratified rates by age showed a rising of ED as the population aged.

Two of the Asian studies showed doubling of the prevalence rate at age 60-70, and almost another doubling at age 70-79 years [15]. A more recent Korean report showed a tripling of the prevalence for the 60-69 age group compared with the younger cohort. In the latter report, this was consistent whether ED was established through self-assessment or by IIEF-5 cut-off scores. Prevalence rate from Australia was overall about 21\% for ages $40-80$ years. For the studies that stratified for age, one study showed an increasing but still low rate of $13-19 \%$ for the decades $40-49$ and 50-59 years. The two other studies stratifying for older ages showed marked increasing rates, starting at age 60 , with increases across each of the three following decades [15]. The most recent report from Western Australia [20] utilized an older age limit in their study and reported an 
overall prevalence of $40 \%$, which corresponds to almost four times the prevalence for the other age-stratified study from Australia which reported an overall rate of $10 \%$.

The prevalence studies from Europe are very difficult to compare. In the Scandinavian studies, prevalence seemed to rise sharply after the age of 65 with rates of $20 \%$ or greater after this age and the rate doubling again when reaching the age of 70 years or older [15]. The prevalence of ED was generally quite low before the age of 61 years in the continental northern European studies. Details of reports from the remaining European studies are presented in the main book chapter and rates vary greatly, particularly for the age group 50-59 years of age. Most of the world showed a rather high rate from $20 \%$ to $40 \%$ for the ages of 60-69 years except for the Scandinavian reports mentioned above. Almost all of the reports showed a high prevalence rate for those men in their $70 \mathrm{~s}$ and 80 s, ranging from $50 \%$ to $75 \%$ in these decades. Below the age of 40 years, the prevalence rate was very low, most below $10 \%$, and continued to be low for the decades 40-49 years of age. There were exceptions for the 40-59 years of age to prevalence rates as high as $20-30 \%$.

As compared with the previous consultation report, there were five new prevalence studies for ED from Latin America for a total of seven reports, six of which came from Brazil. Four random population and community-based population studies reported, with one exception, prevalence rates for ED in the teens before age 50, varying from $12-22 \%$ during the decade $50-59$ and increasing to about $32-58 \%$ in the decades 60-69 years [15]. In the exception study of 2,000 men, prevalence rates were much lower than the other random population studies using the IIEF to define ED from $0.2 \%$ beginning with age 20-40 years of age, $1 \%$ for those $41-50$ years of age, about 3\% for $51-60$ years of age, and $7 \%$ in those greater than 60 years of age [21]. In the seven studies from North America, two studies unstratified for age, reported the prevalence rate for ED of $16 \%$ and $22.5 \%$ [15]. The methods used for both of these studies were very similar and involved 500 men from Canada in one report and 742 men in a report from the United States. For the other five U.S. studies that were stratified for age, prevalence rates for ED were for the most part in the teens for populations aged 18-59 [15]. However, the study from the Massachusetts Male Aging Study showed a prevalence rate of $23 \%$ for ED at age 40 . As observed from the European studies for the 50-59 decades, there was tremendous variance from rates as low as $6-18 \%$ to rates as high as $32-35 \%$ [15]. The rates were much higher for decades 60 and beyond.

In summary, the prevalence of ED on a worldwide basis shows a great deal of variation possibly attributed to the way the information was collected, the way the population was sampled, the tools used for the survey, and more importantly the way ED was defined. Below the age of 40 years, prevalence for $\mathrm{ED}$ is $1-10 \%$. In the decades from 40 to 49 , the prevalence ranges from $2 \%$ to $9 \%$ to as high as $15 \%$. The $50-59$ year age group showed the greatest range of reported prevalence rates. Most of the world showed a rather high rate of $20-40 \%$ for the ages 60-69 years, some increasing after age 65, except for the Scandinavian report where the age of 70 years and older is the decade of major prevalence rate change (there was one report from Finland that showed high rates beginning at age 50) [15]. Almost all of the reports showed a higher prevalence rate for those men in their $70 \mathrm{~s}$ and $80 \mathrm{~s}$ ranging from $50 \%$ to $100 \%$ prevalence of $\mathrm{ED}$ in these decades.

Both in the descriptive and analytical epidemiological literature, there is very little on the simultaneous occurrence of different sexual dysfunctions within and across genders. In French men, aged $18-69$, it has been reported that $7 \%$ of those with manifest ED also are early ejaculators [22]. In an older sample (50-78 years olds) from the Netherlands, about $50 \%$ of the men with early ejaculation also reported ED [23]. To which extent, this simply indicates that the incapacity to maintain erection is a sequel to early ejaculation and remains to be analyzed. From France, it has been reported that $37 \%$ of $18-70$-year-old men with ED have lost their "libido" [24]. It has been found in a Swedish study that within both genders (aged 18-74), nearly all sexual dysfunctions have high within-person correlations [25]. The only exception is the correlation between early and delayed ejaculation, which quite logically was not significant. Moreover, a higher degree of cross-gender concurrence was found. In fact, all women's dysfunctions studied were closely correlated with all male partners' functions/ dysfunctions as perceived by the women. Men's sexual dysfunctions had precisely the same close associations with men's perceptions of their female partner's functions/dysfunctions. These findings firmly suggest that it is important to think in terms of sexual partner relationship, not only for phar- 
macological or psychotherapeutic therapy, but also for assessment. Laumann et al. also examined the co-occurrence of dysfunctions for the set of dysfunctions discussed in the latent class analysis, but the actual percentages of overlap were not given in the report [16].

\section{Risk Factors}

There are common risk factor categories associated with sexual dysfunction for men and women which include the following: general health status of the individual, the presence of diabetes mellitus, the presence of cardiovascular disease, concurrence of other genitourinary disease, psychiatric/ psychological disorders, other chronic diseases, and socio-demographic conditions. For ED, smoking and hormonal factors also serve as welldefined risk factor associated conditions. There is also evidence-based literature for medicationrelated association for ED. Evidence-based criteria should be established for evaluating risk factors for women's and men's sexual dysfunctions.

In women, those who report excellent health compared with good, fair, or poor health are less likely to have a sexual dysfunction. Similarly, women engaging in more physical exercise report higher levels of sexual desire. Also, orgasm problems are more likely among women who reported no physical activity [15]. The previous consultation reported associations of diabetes with low sexual desire, orgasmic dysfunction, dyspareunia, and sexual aversion along with decreased lubrication. However, more recent large epidemiological studies conducted on women in the United States, Brazil, and Australia did not find a significant relationship between diabetes, desire, arousal, or orgasm [15]. In women, hypertension or the use of hypertensive drugs seem to be associated with orgasm and lubrication dysfunction as well as decreased sexual interest. Stress urinary incontinence is negatively associated with all aspects of women's sexual dysfunction (sexual interest, desire, arousal, lubrication, and orgasm), and is also positively correlated with dyspareunia and vaginismus. In a few epidemiological studies, hysterectomy has been found to negatively affect women's sexuality. In women, a psychiatric disorder is closely associated with orgasmic dysfunction and dyspareunia. Cumulating evidence confirm the association between anxiety and depression with sexual dysfunction. Women on different antidepressants have shown negative changes in sexual function. In fact, adverse effects of antidepressants are commonly sexual in nature. Some epidemiological investigations address the impact of sexual abuse on women's sexual behavior and it has been clearly shown that women's current sexual life is negatively correlated with previous abuse [15]. Experiences of emotional and physical abuse during childhood are highly comorbid with a history of sexual abuse and some studies have found initial evidence of a strong relationship between emotional and physical abuse and sexual function as compared with sexual abuse. Being a single woman is associated with dysfunctions of sexual interest, vaginal lubrication, orgasm, and dyspareunia [26], sexual problems that are also more common in women with marital difficulties [15].

Similar to women, less than good overall health is likely to co-occur with men's low level of sexual interest/desire, early ejaculation, and ED. Moreover, men who report to have forced a woman sexually are more likely to have ED than those who have never done so. In a health professional follow-up study, which was controlled for comorbid factors, there was a 10-fold difference in relative risk for $\mathrm{ED}$ associated with older age, regardless of health status or previous ED. Comorbid conditions such as diabetes, cancer, stroke, and hypertension were also associated with an increased risk of ED whereas physical activity, leanness, moderate alcohol consumption, and not smoking were associated with decreased risk [27]. Minority groups are under-represented in most epidemiological studies. In one study, a survey facilitated equivalent representation of nonHispanic whites, non-Hispanic blacks, and Hispanic males 40 years and older. Overall prevalence rates for $\mathrm{ED}$ was $22 \% ; 21 \%$ for whites, $24 \%$ for blacks, and $20 \%$ for Hispanic, all categories increasing with older age. In this report, overall sampled ED probability increased with diabetes, hypertension, and moderate or severe lower urinary tract symptoms [28]. As compared with the previous consultation, it would appear that the predominant evidence available at this time identifies cigarette smoking as an independent risk factor for ED. On the other hand, this committee has not identified descriptive or analytical epidemiological literature which links smoking to other male sexual dysfunctions or to any female sexual dysfunction. In men, diabetes has been associated with a greater prevalence of decreased desire and organic dysfunction as well as ED. Reports of insulin-dependent mellitus, diabetes present for over 10 years, fair or poor control based on glyco- 
sylated hemoglobin, diabetes managed with agents other than diet control, smoking and a history of diabetes mellitus related arterial, renal, or retinal disease and neuropathy show a higher odds ratio for ED. Another major factor documented in the committee's report at this consultation [15], there has been evidence that ED itself could be considered the most efficient predictor of silent coronary artery disease in diabetic populations, independent of glycometabolic control and ED severity [29]. In a very recent study, Rosen et al. showed that diabetics with improved levels of fitness were about $40 \%$ less likely to have ED [30].

In the new consultation chapter, there is a large section devoted to obesity and metabolic syndrome and ED [15]. However, the committee felt that there is still a question whether obesity and the metabolic syndrome per se are additional risk factors for ED or whether cardiovascular disease and diabetes mellitus that are a part of these conditions are the risk factors that account for this association. Further research is needed to answer this question. Endothelial and vascular smooth muscle dysfunction are conditions present in many cases of ED and thus there are common etiological pathways for other vascular disease states, such as cerebral vascular accidents, myocardial infarction, heart disease, hypertension, hyperlipidemia, low serum levels of high density lipoproteins, as well as arteriosclerosis and peripheral vascular disease. Since the last publication of the chapter on risk factor for sexual dysfunction, there has been a stress on the association of cardiovascular disease and ED. It is now thought that ED may be a harbinger or silent coexistent or subsequent coronary artery disease [15]. In a recent cross-sectional observational study, the intimate nexus between ED and cardiovascular disease was presented [31]. In a group of 1,514 men with an age range of 20-99 years, cardiovascular risk factors were more prevalent with increasing age among participants with ED and severe ED. The age adjusted odds of ED were significantly higher among men with hypertension, ischemic heart disease, and stroke. When these three latter conditions were grouped with peripheral arterial disease, the odds ratio also increased for ED. The authors concluded that the relevance and importance of careful cardiovascular risk evaluation in men with ED could not be overemphasized. The committee made a recommendation that although the association of ED and cardiovascular disease and risk factors are well established, there is a need for longitudinal studies in patients who have manifest ED or cardiovascu- lar disease and do not have the opposite manifestassociated condition in order to establish a temporal relationship between the two entities.

The effect androgens on desire/interest and sexual behavior is well established but few reports show direct end organ, corpora cavernosal, dependency on androgens except for early growth and development of the male reproductive tract. Severe hyperprolactinemia (prolactin $>735 \mathrm{mu} / \mathrm{L}$ or $35 \mathrm{ng} / \mathrm{mL}$ ), but not milder forms, has been shown to be associated with decreased sexual desire. Conversely, the impact of high prolactin levels on erectile function is under debate $[15,32]$.

One of the new areas discussed in detail by this committee's book chapter is the association between lower urinary tract symptoms (LUTS) and ED [15]. There has also been an association of LUTS with ejaculatory dysfunction. Several reports have shown that the prevalence of ED and reduced sexual desire, along with other types of sexual dysfunction, is greater in men with LUTS [15]. Moreover, there is a positive correlation between the severity of LUTS and the severity of ED [15]. A recent report was published on the first multinational population-based study to evaluate the relationship among overactive bladder and ED and also sexual quality of life [33]. In this report, those with overactive bladder were significantly more likely to have ED than controls. Other chronic diseases, surgery, and trauma are all described in our chapter as risk factors for ED. Depression has also been closely associated with ED [15]. The committee reviewed extensively medications related to ED and in particular psychiatric drugs are discussed in detail [15].

In the first consultation, modification of risk factors had been reported in one longitudinal study; only increasing physical activity had an impact on incidence data for men in their middle 50s [34]. Since that singular report reported in the first consultation, there has been a recent report by Esposito et al. [35] about the effective and prospective behavior modification in which 209 subjects were randomly assigned to one of two treatment groups. The 104 men randomly assigned to the intervention program received detailed advice about how to reduce body weight, improve quality of diet, and increase physical activity. The control group of 105 subjects was given general information about healthy food choices and general guidance on increasing their level of physical activity. The IIEF-5 scores were similar for groups at the onset of the study. At 
baseline, $35 \%$ of the subjects and $38 \%$ of the control group had normal erectile function. After 2 years, 58 subjects in the intervention group and 40 subjects in the control group showed a significant improvement in erectile function. The conclusion of this study was that it was possible to achieve and improve erectile function in men at risk by means of nonpharmacological intervention aiming at weight loss and increasing physical activity.

Corresponding Author: Ronald W. Lewis, MD, Section of Urology, Medical College of Georgia, BA-8412 Medical College of GA, 1120 15th Street, Augusta, GA 30912-4050, USA. Tel: 706-721-9977; Fax: 706-721-2548; E-mail: rlewis@mcg.edu

Conflict of Interest: There are no conflict of interest to declare for Drs. Lewis, Fugl-Meyer, Corona, Hayes, Laumann, Moreira, Relliini, and Segraves.

\section{References}

1 Summerskill WSM. Hierarchy of evidence. In: McGovern DPB, Summerskill WSM, Valori RM, Levy M, eds. Key topics in evidence based medicine. Oxford: Bios Scientific Publishers; 2001:15-9.

2 Lewis RW, Fugl-Meyer KS, Bosch R, Fugl-Meyer AR, Laumann EO, Lizza E, Martin A. Definitions, classification, and epidemiology of sexual dysfunction. In: Lue TF, Basson R, Rosen R, Giuliano F, Khoury S, Montorsi F, eds. Sexual medicine: Sexual dysfunctions in men and women. Paris: Health Publications; 2004:39-72.

3 Prins J, Blanker MH, Bohnen AM, Thomas S, Bosch JLHR. Prevalence of erectile dysfunction: A systematic review of population based studies. Int J Impot Res 2002;14:42232.

4 McMahon C, Althof S, Waldinger M, Porst H, Dean J, Sharlip I, Adaikan G, Becher E, Broderick G, Buvat J, Dabees K, Giraldi A, Giuliano F, Hellstrom W, Incrocci L. Laan E, Meuleman E, Perelman M, Rosen R, Rowland D, Segraves R. An evidence-based definition of lifelong premature ejaculation: Report of the International Society for Sexual Medicine (ISSM) Ad Hoc Committee for the definition of premature ejaculation. J Sex Med 2008;5:1590-606.

5 Rosen RC, Riley A, Wagner G, Osterloh IH, Kirkpatrick J, Mishra A. The international index of erectile function (IIEF): A multidimensional scale for assessment of erectile dysfunction. Urology 1997;49:822-30.

6 Rosen RC, Brown C, Heiman J, Leiblum SR, Meston C, Shabsigh R, Ferguson D, D'Agostino R. The Female Sexual Function Index (FSFI): A multidimensional self- report instrument for the assessment of female sexual function. J Sex Marital Ther 2000;26:191-208.

7 Hayes RD, Dennerstein L, Bennett CM, Fairley CK. What is the "true" prevalence of female sexual dysfunctions and does the way we assess these conditions have an impact? J Sex Med 2008;5:777-87.

8 Öberg K, Fugl-Meyer AR, Sjögren Fugl-Meyer K. On categorization and quantification of women's sexual dysfunction. An epidemiological approach. Int J Impot Res 2004;16:2619 .
9 Johannes CB, Araujo AB, Feldman HA, Derby CA, Kleinman $\mathrm{KP}, \mathrm{McKinlay} \mathrm{JB}$. Incidence of erectile dysfunction in men 40-69 years old: Longitudinal results from the Massachusetts Male Aging Study. J Urol 2000;163:460-3.

10 Gades NM, Jacobson DJ, McGree ME, St. Sauver JL, Lieber MM, Nehra A, Girman CJ, Jacobsen SJ. Longitudinal evaluation of sexual function in a male cohort: The Olmsted County Study of Urinary Symptoms and Health Status among Men. J Sex Med 2009;6:2455-66.

11 Moreira ED, Lbo CF, Diament A, Nicolosi A, Glasser DB. Incidence of erectile dysfunction in men 40 to 69 years old: Results from a population-based cohort study in Brazil. Urology 2003;61:431-6.

12 Schouten BW, Bosch JL, Bernsen RM, Blanker MH, Thomas $\mathrm{S}$, Bohnen AM. Incidence rates of erectile dysfunction in the Dutch general population. Effects of definition, clinical relevance and duration of follow-up in the Krimpen Study. Int J Impot Res 2005;17:58-62.

13 Shiri R, Koskimäki J, Hakama M, Häkkinen J, Tammela TL, Huhtala H, Auvinen A. Effect of chronic diseases on incidence of erectile dysfunction. Urology 2003;62:1097-102.

14 Shiri R, Koskimäki J, Hakama M, Häkkinen J, Huhtala H, Tammela TL, Auvinen A. Effect of life-style factors on incidence of erectile dysfunction. Int J Impot Res 2004;16:389-94.

15 Fugl-Meyer KS, Lewis RW, Corona G, Hayes RD, Laumann EO, Moreira ED Jr., Rellini AH, Segraves T. Definitions, Classification, and Epidemiology of Sexual Dysfunction. in press.

16 Laumann EO, Paik A, Rosen RC. Sexual dysfunction in the United States: Prevalence and predictors. JAMA 1999;281: 537-44.

17 Nazareth I, Boynton P, King M. Problems with sexual function in people attending London general practitioners: Cross sectional study. BMJ 2003;327:423.

18 Parish WL, Luo Y, Stolzenberg R, Laumann EO, Farrer G, Pan S. Sexual practices and sexual satisfaction: A population based study of Chinese urban adults. Arch Sex Behav 2007;36:5-20.

19 Ahn TJ, Park JK, Lee SW, Hong JH, Park CP, Kim JJ, Park K, Park H, Hyun JS. Prevalence and risk factors for erectile dysfunction in Korean men: Results of an epidemiological study. J Sec Med 2007;4:1269-76.

20 Chew KK, Stuckey B, Bremner A, Earle C, Jamrozik J. Male erectile dysfunction: Its prevalence in Western Australia and associated sociodemographic factors. J Sex Med 2008;5:60-9.

21 De Almeida Claro J, Kaufmann OG, Alarcon G, Agiar W, Nadozza A, Jr., Ortiz V, Srougi M. Could a rural lifestyle decrease the prevalence of erectile dysfunction? BJU Int 2007;99:127-9.

22 Bejin A. Epidemiologie de l'ejaculataion prématurée et de son cumul avec la dysfunction érectile. Andrologie 1999;9:211-25.

23 Blanker MH, Bosch JL, Groeneveld FP, Bohnen AM, Prins A, Thomas S, Hop WC. Erectile and ejaculatory dysfunction in a community-based sample of men 50 to 78 years old: Prevalence, concern, and relation to sexual activity. Urology 2001; 57:763-8.

24 Viraq R, Beck-Ardilly L. Nosologie, épidémiologie, quantification clinique des dysfunctions érectiles. Rev Med Interne 1997;18(1 suppl):10-3.

25 Fugl-Meyer AR, Fugl-Meyer KS. Sexual disabilities are not singularities. Int J Impot Res 2002;14:487-93.

26 Fugl-Meyer AR, Sjögren Fugl-Meyer K. Sexual disabilities, problems and satisfaction in 18-74 year-old Swedes. Scand J Sexol 1999;2:79-105.

27 Bacon CG, Mittleman MA, Kawachi I, Giovannucci E, Glasser D, Rimm EB. Sexual function in men older than 50 years of age: Results from the health professional's follow-up study. Ann Int Med 2003;139:161-8. 
28 Laumann EO, West S, Glasser D, Carson C, Rosen R, Kang J. Prevalence and correlates of erectile dysfunction by race and ethnicity among men aged 40 or older in the United States: From the male attitudes regarding sexual health survey. J Sex Med 2007;4:57-65.

29 Gazzaruso C, Giordanetti S, De Amici E, Bertone G, Falcone C, Geroldi D, Fratino P, Solerte SB, Garzaniti A. Relationship between erectile dysfunction and silent myocardial ischemia in apparently uncomplicated type 2 diabetic patients. Circulation 2004;110:22-6.

30 Rosen RC, Wing RR, Schneider S, Wadden TA, Foster GD, West DS, Kitabchi AE, Brancati FL, Maschak-Carey BJ, Bahnson JL, Lewis CE, Gendrano IN. Erectile dysfunction in type 2 diabetic men: Relationship to exercise fitness and cardiovascular risk factors in the look AHEAD trial. J Sex Med 2009;6:1414-22.

31 Chew KK, Bremner A, Jamrozik K, Earle C, Stuckey B. Male erectile dysfunction and cardiovascular disease: Is there an intimate nexus? J Sex Med 2008;5:928-34.
32 Coronoa G, Mannucci E, Fisher AD, Lotti F, Ricca V, Balercia G, Petrone L, Forti G, Maggi M. Effect of hyperprolactinemia in male patients consulting for sexual dysfunction. J Sex Med 2007;4:1485-93.

33 Irwin DE, Milson I, Reilly K, Hunskaar S, Kipp Z, Herschorn S, Coyne KS, Kelleher CJ, Artibani W, Abrams P. Overactive bladder is associated with erectile dysfunction and reduced sexual quality of life in men. J Sex Med 2008;5:2904-10.

34 Derby CA, Mohr B, Goldstein I, Feldman HA, Johannes CB, McKinlay JB. Modifiable risk factors and erectile dysfunction: Can life-style changes modify risk? Urol 2000;56: 302-6.

35 Esposito K, Ciotola M, Giugliano F, Maiorino MI, Autorino R, De Sio M, Biugliano G, Nicoletti G, D’Andrea F, Giugliano D. Effects of intensive lifestyle changes on erectile dysfunction in men. J Sex Med 2009;6:243-50. 